\title{
S07-01
}

\section{CLINICAL AND EPIDEMIOLOGICAL DATA ON EATING DISORDERS NOS}

\section{P. Machado}

Psychology, Universidade do Minho, Braga, Portugal

Eating Disorders Not Otherwise Specified (EDNOS) represent the most common eating disorder diagnosed in specialized treatment settings. The purpose of the current presentation is to review epidemiological data on this residual diagnostic category. Namely we will present data on the prevalence of EDNOS in a nationwide community sample. In this study, participants were 2028 female students, aged 12 to 23, attending public schools in the 9th to 12th grades in Portugal. Participants completed the Eating Disorder Examination Questionnaire in Stage 1 of the study. In Stage 2, we selected all the participants who met any of these criteria:

1. $\mathrm{BMI} \leq 17.5$,

2. scores $\geq 4$ on any of the four EDE-Q Subscales,

3. a total EDE-Q score $\geq 4$, or,

4. the presence of dysfunctional eating behaviors.

In Stage 2, eating disorder experts interviewed 901 participants using the Eating Disorder Examination. The prevalence of all eating disorders was $3.06 \%$ among young females. Prevalence for anorexia nervosa was $0.39 \%$, for bulimia nervosa $0.30 \%$, EDNOS $2.37 \%$. EDNOS was a very common eating disorder and accounts for three-quarters of all community cases with eating disorders. Implications for research and treatment will be presented. 